\title{
Questionnaire Survey of Patients with Type-1 Diabetes Mellitus and their Family Members on the Acceptance of Newly Emerging Therapies
}

\section{Masayuki Shimoda* and Shinichi Matsumoto}

Islet Cell Transplantation Project, Diabetes Research Center, Research Institute of National Center for Global Health and Medicine, Tokyo, Japan

\begin{abstract}
Objective: A current goal for the treatment of Type 1 Diabetes Mellitus (T1DM), an incurable disease, is to prevent deterioration of patients' Quality Of Life (QOL) by maintaining excellent glycemic control. Health care providers expect that newly developed therapies will help improve QOL, but QOL must be assessed by the patients themselves. Therefore, it is critical to understand patients' opinions about newly developed therapies. The aim of this study was to investigate patients' opinions, especially their motivation to accept newly developed therapies.

Method: We analyzed questionnaires $(n=85)$ completed by T1DM patients and family members about four newly developed therapies-Allogeneic Islet Transplantation (AIT), Islet Xenotransplantation (XIT), DNA vaccination, and Induced Pluripotent Stem cell therapy (IPS)-to investigate the factors in their acceptance of these therapies.

Results: A total of $56.3 \%$ of patients and $74.8 \%$ of family members accepted the newly developed therapies. Experience of hypoglycemic events, the main indication for AIT, did not significantly influence acceptance of the newly developed therapies. Desire to be insulin free had significant influence on the acceptance of AIT and IPS $(p<0.05$, respectively).
\end{abstract}

Conclusion: Achieving insulin-free status is a more important motivator than avoiding hypoglycemia for patients to accept newly developed therapies.

Keywords: Allogeneic islet transplantation; Islet xenotransplantation; DNA vaccination; Induced pluripotent stem cell therapy; Quality of life

\section{Introduction}

A major goal in current treatments for Type 1 Diabetes Mellitus (T1DM), an incurable disease, is to prevent deterioration of patients' Quality of Life (QOL) by maintaining excellent glycemic control. To this end, we performed the world's first living donor pancreatic islet transplantation and allogeneic islet transplantation from cadaver donors in T1DM patients [1-3]. From these experiences, we have realized that the importance of patients' self-assessments of treatments is high, because QOL can be determined by only the patients themselves. We therefore believe that patients' self-assessments should be given due consideration in regard to newly developed therapies.

Allogeneic Pancreatic Islet Transplantation (AIT), one of the therapies recently developed for treating T1DM, has already been used in the treatment of T1DM patients worldwide [4,5]. However, although it is clear that AIT is a minimally invasive and effective betacell replacement therapy [5], a number of problems still need to be resolved to expand its application. These problems include a shortage of organ donors and the necessity of immunosuppressive drugs to prevent both allogeneic rejection and autoimmune recurrence. To overcome such problems, several novel therapies are under development. Among them, islet transplantation using encapsulated porcine islets (XIT) and human induced pluripotent stem cell-derived islet-like cells (IPS) could overcome the problems posed by donor shortage. A gene vaccine therapy (DNA vaccination, DNA), which focuses on preventing autoimmune attack of beta cells, is also under development. Among these newly developed treatments, AIT [4,5], XIT [6], and DNA [7] have been clinically applied, whereas IPS remains at a basic research stage.

Health care providers expect that newly developed therapies will solve current problems and improve patients' QOL. However, QOL must be assessed by the patients themselves $[8,9]$. Therefore, it is important to understand patients' opinions about newly developed therapies.

The aim of this study is to investigate the opinions of T1DM patients and their family members, especially regarding their acceptance of the four abovementioned newly developed therapies.

\section{Materials and Methods}

\section{Ethical guidelines}

The institutional ethics committee approved this survey study and the questionnaire respondents provided written informed consent to participate in the study. For respondents who were adolescents, their parents provided written consent.

\section{Study population}

At a symposium held by a T1DM patient group in March 2013, participants attended lectures about AIT, XIT, DNA, and IPS presented by experts on each topic. Table 1 shows the main topics presented in the lectures. Patients completed questionnaires at the conclusion of the four lectures. The respondents in this study included some adolescent T1DM patients, even though islet transplantation is available for only

*Corresponding author: Masayuki Shimoda, Islet Cell Transplantation Project, Diabetes Research Center, Research Institute of National Center for Global Health and Medicine, Tokyo, Japan, Tel: +81-3-3202-7181; E-mail: mshimoda@hosp.ncgm.go.jp

Received June 28, 2014; Accepted September 26, 2014; Published October 06 2014

Citation: Shimoda M, Matsumoto S (2014) Questionnaire Survey of Patients with Type-1 Diabetes Mellitus and their Family Members on the Acceptance of Newly Emerging Therapies. J Diabetes Metab 5: 442 doi:10.4172/2155-6156.1000442

Copyright: @ 2014 Shimoda M, et al. This is an open-access article distributed under the terms of the Creative Commons Attribution License, which permits unrestricted use, distribution, and reproduction in any medium, provided the original author and source are credited. 
Citation: Shimoda M, Matsumoto S (2014) Questionnaire Survey of Patients with Type-1 Diabetes Mellitus and their Family Members on the Acceptance of Newly Emerging Therapies. J Diabetes Metab 5: 442 doi:10.4172/2155-6156.1000442

Page 2 of 5

adults in Japan, because adolescents give serious consideration to their disease and might become candidates for the new therapies in the future.

\section{Opinion survey and study variables}

Transplant surgeons, diabetologists, and expert data analysts validated the contents of the answered questionnaires. The questionnaire comprised questions on basic characteristics, frequency of hypoglycemic events, desire to be insulin free, and willingness to receive the newly developed therapies AIT, XIT, DNA, and IPS. Three types of hypoglycemic events were defined: hypoglycemic coma (COMA), severe hypoglycemia requiring a third party's help (HELP), and Unaware Hypoglycemia (UHG). For each question, respondents selected one of four options according to their level of desire or agreement (strongly wish/agree, wish/agree, do not wish/do not agree, and strongly do not wish/strongly do not agree). Four options were stratified into two groups: the wish/accept group (wish/accept, strongly wish/strongly accept) and the do not wish/do not accept group (do not wish/do not accept, strongly do not wish/strongly do not accept).

We analyzed acceptance rates of the newly developed treatments based on satisfaction with current treatments, desire to be insulin free, and experience of hypoglycemic events. We also analyzed discrepancies between the patients and their family members.

\section{Statistical Analysis}

Data were analyzed using descriptive statistics and Fisher's exact test. Differences were considered significant at $\mathrm{P}<0.05$.

\section{Results}

From the 105 questionnaires collected, 85 questionnaires (53 from patients, 32 from family members) were qualified for analysis. Table 2 shows the participants' characteristics. More than $60 \%$ of patients had experienced some type of hypoglycemic event.

Although more than $70 \%$ of patients were satisfied with their current insulin treatment, more than $90 \%$ of patients wished to be insulin free (Table 3). Notably, $100 \%$ of family members wanted patients to be insulin free.

More than $40 \%$ of patients and more than $60 \%$ of family members accepted all four newly developed therapies. In general, family members had higher acceptance rates than patients for all newly developed therapies; in particular, they had significantly higher acceptance rates of XIT and IPS (Table 3). Age of respondents and duration of T1DM had no influence on acceptance of the new therapies.

Patients' satisfaction with their current treatment did not have a significant influence on their acceptance of any of the newly developed

\begin{tabular}{|c|c|c|}
\hline Therapy & Advantages & Disadvantages \\
\hline AIT & $\begin{array}{c}\text { Achieve excellent glycemic control without hypoglycemia } \\
\text { Possible to achieve insulin-free status } \\
\text { Minimally invasive transplant } \\
\text { Many clinical cases }\end{array}$ & $\begin{array}{c}\text { Donor shortage } \\
\text { Expensive } \\
\text { Need for immunosuppression } \\
\text { Poor long-term efficacy for insulin independence }\end{array}$ \\
\hline XIT & $\begin{array}{c}\text { Reduced occurrence of unaware hypoglycemia } \\
\text { No immunosuppression with encapsulation technology } \\
\text { No donor shortage } \\
\text { Sterile donor pigs }\end{array}$ & $\begin{array}{l}\text { Limited clinical experience } \\
\text { Low efficacy compared to AIT at present } \\
\text { Possible zoonosis from pig islets }\end{array}$ \\
\hline DNA & $\begin{array}{l}\text { Prevent progression of type } 1 \text { diabetes by suppressing auto-immune } \\
\text { activation }\end{array}$ & $\begin{array}{l}\text { Recent clinical trial using GAD65 vaccination was not so effective } \\
\text { Beta-cell replacement therapy is needed once patients lose the majority of islets }\end{array}$ \\
\hline IPS & $\begin{array}{c}\text { Possible to create beta cells } \\
\text { Infinite cell expansion } \\
\text { Japan leads this field }\end{array}$ & $\begin{array}{c}\text { Still experimental } \\
\text { Technical difficulty creating beta cells }\end{array}$ \\
\hline
\end{tabular}

Note. AIT = Allogeneic Islet Transplantation; XIT = Islet Xenotransplantation; DNA = DNA Vaccination; IPS = Induced Pluripotent Stem Cell Therapy.

Table 1: Main topics covered in the lecture on each therapy's advantages and disadvantages.

\begin{tabular}{|c|c|}
\hline & Total $(n=85)$ \\
\hline \multicolumn{2}{|l|}{ Standpoint } \\
\hline Patient & $53(62 \%)$ \\
\hline Family & $32(38 \%)$ \\
\hline Age of patient (year) (range) & $32.7 \pm 16.0(3-74)$ \\
\hline Age of family member (year) (range) & $51.0 \pm 8.0(30-69)$ \\
\hline \multicolumn{2}{|l|}{ Gender of patient } \\
\hline Male & $25(29 \%)$ \\
\hline Female & $60(71 \%)$ \\
\hline Onset age (range) & $22.0 \pm 15.8(1-67)$ \\
\hline Disease duration (year) (range) & $11.0 \pm 11.8(0-45)$ \\
\hline Experience of unconscious hypoglycemic attack & $14(17 \%)$ \\
\hline Mean number of unconscious hypoglycemic attacks per year (range) & $1.5 \pm 10.9(0-100)$ \\
\hline Experience of hypoglycemic attacks requiring other's help & $23(28 \%)$ \\
\hline Mean number of hypoglycemic attacks requiring another's help per month (range) & $0.4 \pm 0.9(0-4)$ \\
\hline Experience of unaware hypoglycemia & $46(55 \%)$ \\
\hline Mean number of unaware hypoglycemia incidents per week (range) & $1.0 \pm 1.9(0-10)$ \\
\hline
\end{tabular}

Table 2: Characteristics of participants. 
Citation: Shimoda M, Matsumoto S (2014) Questionnaire Survey of Patients with Type-1 Diabetes Mellitus and their Family Members on the Acceptance of Newly Emerging Therapies. J Diabetes Metab 5: 442 doi:10.4172/2155-6156.1000442

Page 3 of 5

\begin{tabular}{|c|c|c|c|c|}
\hline & Total $(N=85)$ & Patient $(n=53)$ & Family $(n=32)$ & $P$-value (Patient:Family) \\
\hline I am satisfied with the current treatment. & $60(73.2 \%)$ & $37(71.2 \%)$ & $23(76.7 \%)$ & 0.80 \\
\hline I wish to be insulin free. / wish the patient to be insulin free. & $81(95.3 \%)$ & $49(92.5 \%)$ & $32(100 \%)$ & 0.29 \\
\hline I wish to receive AIT/I wish the patient to receive AIT. & $42(56.8 \%)$ & $25(54.3 \%)$ & $17(60.7 \%)$ & 0.64 \\
\hline I wish to receive XIT/I wish the patient to receive XIT. & $42(60.9 \%)$ & $24(52.2 \%)$ & $18(78.3 \%)$ & $<0.05$ \\
\hline I wish to receive DNA vaccination/I wish the patient to receive DNA vaccination. & $35(49.3 \%)$ & $19(41.3 \%)$ & $16(64.0 \%)$ & 0.09 \\
\hline I wish to receive IPS/I wish the patient to receive IPS. & $59(84.3 \%)$ & $34(77.3 \%)$ & $25(96.2 \%)$ & $<0.05$ \\
\hline
\end{tabular}

Note. Values are expressed as the number (percentage) of wish/agree to the questions. $P$-value shows the probability of significant differences between Patient group and Family group. AIT = Allogeneic Islet Transplantation; XIT = Islet Xenotransplantation; IPS = Induced Pluripotent Stem Cell Therapy.

Table 3: Results of questionnaire items.

A

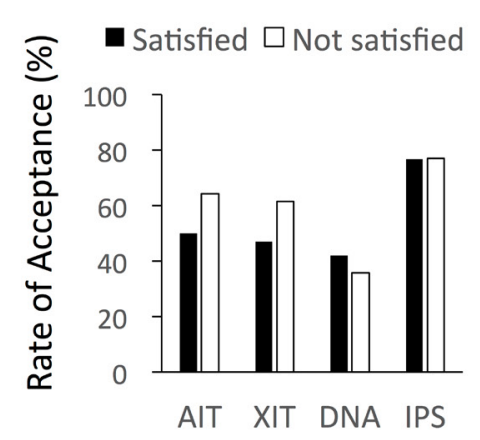

B

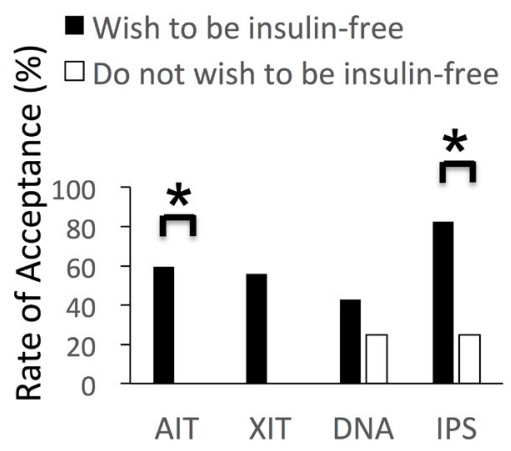

C

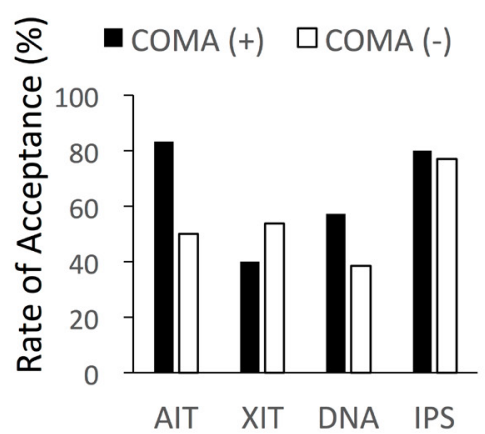

D

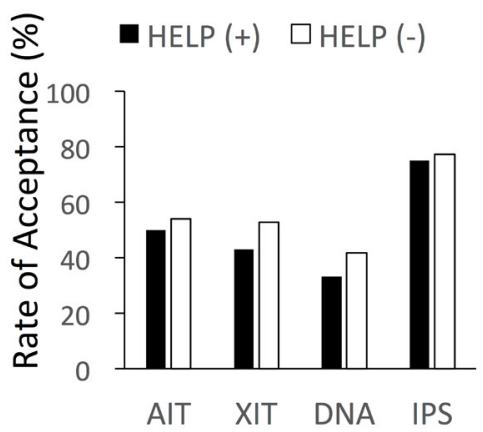

E

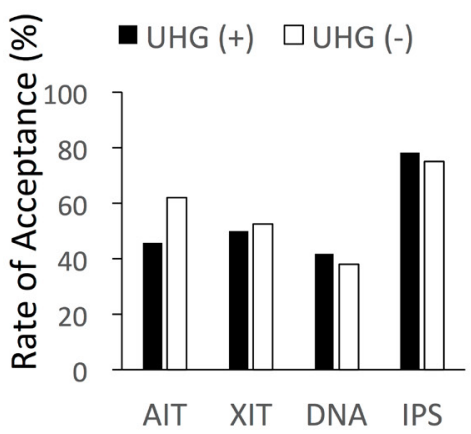

Figure 1: Variables related to acceptance among type 1 diabetes patients of new therapies

Comparison of the percentage of patients who expressed willingness to receive new therapies based on several motivational factors. Patients were divided into two groups based on whether they (A) were satisfied with the current treatment or not, (B) wished to be insulin free or not, (C) had experienced an unconscious hypoglycemic attack (COMA+) or not (COMA-), (D) had experienced severe hypoglycemia requiring a third party's help (HELP+) or not (HELP-), and (E) had experienced unaware hypoglycemia $(\mathrm{UHG}+)$ or not (UHG-). AIT = Allogeneic Islet Transplantation; XIT = Islet Xenotransplantation; DNA = DNA Vaccination; IPS = Induced Pluripotent Stem Cell Therapy. ${ }^{*} P$-value $<0.05$ between the two groups.

therapies (Figure 1A). In the patients who wished to be insulin free, the rates of acceptance of AIT, XIT, DNA, and IPS were 59.5\%, 55.8\%, $42.9 \%$, and $82.5 \%$, respectively (Figure 1B). The rates of acceptance of AIT and IPS were significantly higher in the patients who wished to be insulin free than in the patients who did not $(\mathrm{p}<0.05$, respectively). XIT showed the same trend, but did not reach statistical significance (Figure 1B).

More than $80 \%$ of patients who experienced COMA but less than $50 \%$ of patients who did not accepted AIT (Figure 1C). Therefore, COMA experience could be a motivation to accept AIT, although it did not reach statistical significance (Figure 1C). Except for COMA, experience of hypoglycemic events did not influence the acceptance of any of the newly developed therapies (Figure 1C-E).

\section{Discussion}

In this study, we conducted a questionnaire survey about newly developed therapies for T1DM immediately after patients and their family members had attended the lectures about the disease. Therefore, the lecture contents clearly influenced the survey outcomes.

In this study, we found that more than $25 \%$ of patients were dissatisfied with their current treatment, a result similar to that found in our previous survey [8]. Furthermore, more than 50\% of patients and their family members accepted all four newly developed therapies. These findings suggest that research on newly developed therapies for the treatment of T1DM is important from the patients' viewpoint. 
Interestingly, for all therapies, the acceptance rates of the family members were higher than those of the patients. This is probably because the majority of the family members were likely the patients' parents. Therefore, they would naturally worry about them and might pay greater attention to the benefits of the therapies rather than the risks. Conversely, the patients themselves might pay more attention to the risks. It is important to recognize that the opinions about newly developed therapies are different between the two groups. Accordingly, a newly developed therapy should be started with only adults who have established autonomy.

In terms of AIT, a University of Edmonton group in 2000 demonstrated that 7 of 7 patients became insulin free after undergoing multiple AIT (the Edmonton protocol) [4]. Five years after this announcement, less than $10 \%$ of the AIT patients were able to maintain their insulin-free status; however, more than $80 \%$ of the AIT patients were able to maintain excellent glycemic control even after resuming insulin injections [10]. Excellent glycemic control leads to avoidance of hypoglycemia, which in turn improves patients' QOL. Therefore, the main purpose of AIT was switched from achieving insulin-free status to avoiding hypoglycemia. Single donor islet transplantation should be able to achieve this goal with the current advanced technology for islet isolation and transplantation [3,11]. Islet transplantation with a sufficient amount of islet cells and supplemental islet transplantation should achieve an insulin-free status over the long term [12-14]. However, both strategies require more donor pancreata, which could worsen the donor shortage. The dilemma is whether AIT should focus on achieving insulin-free status even though it requires more donors, or on maintaining excellent glycemic control to avoid hypoglycemic events using a minimal number of donors.

In this study, approximately $60 \%$ of patients who wished to be insulin free accepted AIT, whereas none of the 4 patients who did not wish to be insulin free accepted it. Therefore, even though the primary endpoint of current AIT is avoiding hypoglycemia, becoming insulin free appears to be an important goal of AIT for T1DM patients. On the other hand, over $80 \%$ of patients who had experienced coma accepted AIT, suggesting that experiencing hypoglycemic coma can be a motivator for accepting AIT. When we consider together all factors from the patients' viewpoint, if there are enough donors we should aim for insulin-free status; otherwise, we should focus on rescuing patients from the fear of hypoglycemia, especially coma. These thoughts differ from those of diabetologists, who seek to apply AIT to provide excellent glycemic control and prevent hypoglycemia (not only coma but also severe hypoglycemia and unaware hypoglycemia) [11]. If we could overcome the donor shortage, insulin independence could be achieved by providing sufficient islets, which would satisfy both patients and diabetologists.

XIT has been conducted to overcome the donor shortage [6]. The major hurdles of xenotransplantation are immunological rejection and potential viral infection. Recently, we reported on clinical XIT performed under a comprehensive regulatory framework in New Zealand [15]. This framework minimizes the infectious risk by using a designated pathogen-free herd, current good manufacturing practice for islet isolation and encapsulation, and a comprehensive monitoring system for patients and their close contacts. Encapsulation technology can create an immune-privileged site to prevent immunorejection without the need for immunosuppression. Avoiding immunosuppression is beneficial to the patients by eliminating its side effects and by maintaining the immune system to minimize the risk of infection. On the other hand, current encapsulation technology can also deteriorate islet function. Therefore, the main endpoint of the New Zealand trial was to reduce cases of unaware hypoglycemia rather than to achieve insulin-free status [15]. The International Xenotransplantation Association recommended that an acceptable profile for T1DM patients to receive islet xenotransplantation is having either unaware hypoglycemia that could lead to potentially fatal severe hypoglycemia or immunosuppression because of kidney transplantation [16]. Therefore, reducing occurrences of unaware hypoglycemia seems a reasonable indication for XIT from the doctor's viewpoint. In this study, more than $50 \%$ of patients who wished to be insulin free accepted XIT, whereas none of the patients who did not wish to be insulin free accepted this treatment. Even reducing cases of unaware hypoglycemia is important for improving patients' QOL, and achieving insulin-free status seems to be an important endpoint for patients.

In terms of DNA, acceptance was not positively influenced by any factor, including satisfaction with current treatment, the wish to be insulin free, or experience of hypoglycemic events. This is probably because DNA is mainly used to prevent the onset of type 1 diabetes. In fact, the lecture contents on DNA for participants mentioned that patients might need beta-cell replacement therapy in addition to DNA. This might be why patients and their family members did not seem so interested in this therapy.

Among all of the four newly developed therapies that we focused on, IPS had the highest acceptance rate. Interestingly, only this treatment out of the four considered has never been applied clinically and has never been the subject of any pre-clinical large animal studies. The lecture contents on IPS explained that this therapy could potentially cure T1DM without immunosuppression if new beta cells were created from the patients' own cells. Because clinical and pre-clinical data are lacking, it was not possible to consider side effects or adverse events linked with IPS. Thus, patients might have appreciated the benefits of this "dream" treatment without hearing about specific drawbacks. However, problems with this dream treatment are now becoming apparent. For example, creating beta cells from a patient's own cells is laborious and expensive. Instead of using the patient's own cells, a human leukocyte antigen haplotype-based IPS cell bank is being established to reduce immunogenicity, but this strategy still requires immunosuppression therapy [17]. Risks of tumorigenesis might become apparent once this treatment gets closer to a clinical trial. It is critically important to provide appropriate information on both the potential benefits and risks of therapy for making appropriate decisions.

The newly developed therapies differ in many regards, such as the current status of development, effects, risks, and complications, necessity of donors, opportunities, and costs. Therefore, it is hard to compare the therapies simply and the results should be interpreted carefully. Nevertheless, this study can offer important information to researchers and medical providers, because the survey was answered entirely by T1DM patients and their family members regarding the newly developed therapies and this type of study has only rarely been performed. Although not contained in this questionnaire, a respondent's detailed feedback may be useful for investigating the relationship between acceptance of the new therapies and patients' and family members' characteristics, for example, education level, relationship to the patient, glycemic control (as assessed using general indicator such as HbA1c), and detailed T1DM classification. Further research should focus on these topics.

In conclusion, more than half of T1DM patients and their families accepted the newly developed therapies. On the other hand, the current main endpoint of AIT such as avoiding hypoglycemia might not be 
Citation: Shimoda M, Matsumoto S (2014) Questionnaire Survey of Patients with Type-1 Diabetes Mellitus and their Family Members on the Acceptance of Newly Emerging Therapies. J Diabetes Metab 5: 442 doi:10.4172/2155-6156.1000442

Page 5 of 5

attractive enough to motivate patients to accept the newly developed therapies. The experiences of severe hypoglycemic events such as coma could drive an increase in acceptance of these therapies. Finally, insulin-free status is an important goal for patients' acceptance of the new therapies.

\section{Acknowledgements}

The authors thank Yukio Ohsawa and colleagues (School of Engineering, The University of Tokyo) and Mieko Kishimoto (National Center for Global Health and Medicine) for their support, and thank the non-profit organization, Japan IDDM Network, for its cooperation.

\section{Conflict of interest}

MS was partially supported by grants from the Japan IDDM Network and Japan Science and Technology Agency (Research Center Network for Realization of Regenerative Medicine). SM is a senior advisor to Otsuka Pharmaceutical Factory, Inc. and a director of Diatranz Otsuka Ltd.

\section{References}

1. Matsumoto $S$, Okitsu T, Iwanaga $Y$, Noguchi $H$, Nagata $H$, et al. (2006) Successful islet transplantation from non-heart-beating donor pancreata using modified Ricordi islet isolation method. Transplantation 82: 460-465.

2. Matsumoto S, Okitsu T, Iwanaga Y, Noguchi H, Nagata H, et al. (2005) Insulin independence after living-donor distal pancreatectomy and islet allotransplantation. Lancet 365: 1642-1644.

3. Matsumoto S, Takita M, Chaussabel D, Noguchi H, Shimoda M, et al. (2011) Improving efficacy of clinical islet transplantation with iodixanol-based islet purification, thymoglobulin induction, and blockage of IL-1 $\hat{\mid}^{2}$ and TNF-î̀. Cell Transplant 20: 1641-1647.

4. Shapiro AM, Lakey JR, Ryan EA, Korbutt GS, Toth E, et al. (2000) Islet transplantation in seven patients with type 1 diabetes mellitus using a glucocorticoid-free immunosuppressive regimen. N Engl J Med 343: 230-238.

5. Matsumoto S (2010) Islet cell transplantation for Type 1 diabetes. J Diabetes 2: $16-22$.

6. Elliott RB, Escobar L, Tan PL, Muzina M, Zwain S, et al. (2007) Live encapsulated porcine islets from a type 1 diabetic patient $9.5 \mathrm{yr}$ after xenotransplantation. Xenotransplantation 14: 157-161.

7. Krause S, Landherr U, Agardh CD, Hausmann S, Link K, et al. (2014) GAD autoantibody affinity in adult patients with latent autoimmune diabetes, the study participants of a GAD65 vaccination trial. Diabetes Care 37: 1675-1680.

8. Hatanaka N, Takita M, Yamaguchi T, Kami M, Matsumoto S (2010) Interests in beta-cell replacement therapies among Japanese patients with type 1 diabetes. Diabetes Res Clin Pract 89: e5-8.

9. Speight J, Reaney MD, Woodcock AJ, Smith RM, Shaw JA (2010) Patientreported outcomes following islet cell or pancreas transplantation (alone or after kidney) in Type 1 diabetes: a systematic review. Diabet Med 27: 812-822.

10. Ryan EA, Paty BW, Senior PA, Bigam D, Alfadhli E, et al. (2005) Five-year follow-up after clinical islet transplantation. Diabetes 54: 2060-2069.

11. Sassa M, Fukuda K, Fujimoto S, Toyoda K, Fujita $Y$, et al. (2006) A single transplantation of the islets can produce glycemic stability and reduction of basal insulin requirement. Diabetes Res Clin Pract 73: 235-240.

12. Matsumoto S, Noguchi H, Takita M, Shimoda M, Tamura $Y$, et al. (2010) Super-high-dose islet transplantation is associated with high SUITO index and prolonged insulin independence: a case report. Transplant Proc 42: 2156-2158.

13. Matsumoto S, Takita M, Shimoda M, Chujo D, Itoh T, et al. (2011) Insulin independence by supplemental islet transplantation 5 years after initial islet transplantation. J Diabetes 3: 353-355.

14. Koh A, Imes S, Kin T, Dinyari P, Malcolm A, et al. (2010) Supplemental islet infusions restore insulin independence after graft dysfunction in islet transplant recipients. Transplantation 89: 361-365.

15. Matsumoto S, Tan P, Baker J, Durbin K, Tomiya M, et al. (2014) Clinical porcine islet xenotransplantation under comprehensive regulation. Transplant Proc 46: 1992-1995.

16. O'Connell PJ (2009) The International Xenotransplantation Association consensus statement on conditions for undertaking clinical trials of porcine islet products in type 1 diabetes--chapter 6: Patient selection for pilot clinical trials of islet xenotransplantation. Xenotransplantation 16: 249-254

17. Zimmermann A, Preynat-Seauve O, Tiercy JM, Krause KH, Villard J (2012) Haplotype-based banking of human pluripotent stem cells for transplantation potential and limitations. Stem Cells Dev 21: 2364-2373. 